\title{
An Update of the Transfusion-Related Acute Lung Injury (TRALI): A Proposed Modified Definition and Classification Scheme Definition
}

\author{
Alexander P. J. Vlaar ${ }^{1,2} \cdot$ Steve Kleinman ${ }^{3}$
}

Received: 26 July 2019/ Accepted: 27 August 2019/Published online: 3 September 2019

(C) Indian Society of Hematology and Blood Transfusion 2019

\section{To the editor}

Transfusion-related acute lung injury (TRALI) is among the leading causes of transfusion-related morbidity and mortality. Over the past 20 years, insight into TRALI pathogenesis has resulted in implementation of preventive strategies both from the transfusion medicine/blood bank and clinical settings. Based on this accumulated clinical and basic science knowledge, a panel of 10 international experts on TRALI, including two members with haemovigilance expertise, used the Delphi approach to develop a recently published redefinition of TRALI by modifying the widely used 2004 TRALI Canadian Consensus Conference (CCC) definition (Table 1) [1]

The updated TRALI definition/classification scheme along with the rationale for the changes has now been published (Table 2) [2]. The main modifications are: (1) TRALI has been separated into two types. TRALI type I applies to cases without an ARDS risk factor and TRALI type II to cases with an ARDS risk factor or with mild pre-existing ARDS. Notably, the presence of either an ARDS risk factor or mild ARDS does not exclude the diagnosis of TRALI as it did under the old definition; (2) the term "possible TRALI" has been dropped; (3) Cases with an ARDS risk factor which meet ARDS diagnostic criteria and where respiratory deterioration over the $12 \mathrm{~h}$ prior

Alexander P. J. Vlaar

a.p.vlaar@amc.uva.nl

1 Department of Intensive Care Medicine, Academic Medical Center, Room, C3-430, Meibergdreef 9,

1105 AZ Amsterdam, The Netherlands

2 Laboratory of Experimental Intensive Care and Anesthesiology (L.E.I.C.A.), Academic Medical Center, Amsterdam, The Netherlands

3 Department of Pathology, University of British Columbia, Vancouver, Canada to transfusion implicates the risk factor as causative should be classified as ARDS rather than TRALI type II; (4) The 2012 updated ARDS consensus definition (referred to as the BERLIN ARDS definition) has been evaluated for its relevance to TRALI and essential updates (including updating the list of commonly accepted ARDS risk factors and expanding the methods for diagnosing hydrostatic pulmonary edema) have been incorporated into the new TRALI definition.

More broadly, the Delphi panel recommended that all pulmonary complications after blood transfusion should be reported to the transfusion service and then categorized (either by the transfusion service, a hospital transfusion committee or a haemovigilance system) into one of several categories: TRALI (type I or type II), ARDS, transfusion associated circulatory overload (TACO), TRALI/TACO cannot distinguish, or an alternate diagnosis. Importantly, the panel reaffirmed that TRALI remains a clinical diagnosis and does not require detection of cognate leukocyte antibodies, though it did recommend that these data be captured through a haemovigilance reporting system. Future research directions have been identified and include identifying the mechanism behind the onset of TRALI in the absence of cognate leukocyte antibodies. Furthermore, the panel is working on developing a universal reporting form for post-transfusion pulmonary complications including suspected TRALI.

We believe that the modified TRALI definition is of substantial importance and needs to be widely disseminated and discussed. To this end, the panel has submitted similar letters to the editor of several important transfusion and hemovigilance journals [3, 4]. In addition, a revised surveillance definition of Transfusion Associated Circulatory Overload (TACO) has recently been published [5]. We hope that these new definitions will contribute to an enhanced level of reporting and a more accurate classification of respiratory complications associated with blood transfusion. 
Table 12004 Canadian Consensus Conference definition of TRALI and possible TRALI [1]

\begin{tabular}{|c|c|c|c|}
\hline \multirow[t]{14}{*}{ 1. TRALI } & \multirow[t]{11}{*}{ (a) } & (i) & Acute onset \\
\hline & & (ii) & Hypoxemia \\
\hline & & & Research setting \\
\hline & & & $\mathrm{PaO}_{2} / \mathrm{F}_{\mathrm{i}} \mathrm{O}_{2} \leq 300$ \\
\hline & & & or $\mathrm{SpO}_{2}<90 \%$ on room air \\
\hline & & & Non-research setting \\
\hline & & & $\mathrm{PaO}_{2} / \mathrm{F}_{\mathrm{i}} \mathrm{O}_{2} \leq 300$ \\
\hline & & & or $\mathrm{SpO}_{2}<90 \%$ on room air \\
\hline & & & or other clinical evidence of hypoxemia \\
\hline & & (iii) & Bilateral infiltrates on chest radiograph \\
\hline & & (iv) & $\begin{array}{l}\text { No evidence of left atrial hypertension and/or } \\
\text { central venous pressure }<18 \mathrm{mmHg}\end{array}$ \\
\hline & (b) & \multicolumn{2}{|c|}{ No preexisting ALI before transfusion } \\
\hline & (c) & \multicolumn{2}{|c|}{ During or within $6 \mathrm{~h}$ of transfusion } \\
\hline & (d) & \multicolumn{2}{|c|}{ No temporal relationship to an alternative risk factor for ALI } \\
\hline \multirow[t]{2}{*}{ 2. Possible TRALI } & (a) & \multicolumn{2}{|c|}{ As mentioned above } \\
\hline & (b) & \multicolumn{2}{|c|}{ In the presence of an alternative risk factor for ALI } \\
\hline
\end{tabular}

Table 2 New consensus TRALI definition [2]

TRALI type I: Patients who have no risk factors for ARDS and meet the following criteria

(a) (i) Acute onset

(ii) Hypoxemia

$$
\begin{aligned}
& \mathrm{PaO}_{2} / \mathrm{F}_{\mathrm{i}} \mathrm{O}_{2} \leq 300^{\mathrm{a}} \\
& \text { or } \mathrm{SpO}_{2}<90 \% \text { on room air }
\end{aligned}
$$

(iii) Clear evidence of bilateral pulmonary edema on imaging (e.g. chest radiograph, chest CT, or ultrasound)

(iv) No evidence of left atrial hypertension $(\mathrm{LAH})^{\mathrm{b}}$ or, if LAH is present, it is judged to not be the main contributor to the hypoxemia

(b) Onset during or within $6 \mathrm{~h}$ of transfusion ${ }^{\mathrm{c}}$

(c) No temporal relationship to an alternative risk factor for ARDS

TRALI type II: Patients who have risk factors for ARDS (but who have not been diagnosed with ARDS) or who have pre-existing mild ARDS $\left(\mathrm{PaO}_{2} / \mathrm{F}_{i} \mathrm{O}_{2}\right.$ of 200-300), but whose respiratory status deteriorates ${ }^{\mathrm{d}}$ and is judged to be due to transfusion based

(a)

(b)
Findings as described in categories $a$ and $b$ of TRALI type I, and

Stable respiratory status in the $12 \mathrm{~h}$ prior to transfusion

${ }^{a}$ If altitude is higher than $1000 \mathrm{~m}$, the correction factor should be calculated as follows: $\left[\left(\mathrm{PaO}_{2} / \mathrm{FiO}_{2}\right) \times(\right.$ barometric pressure/760)]

${ }^{\mathrm{b}}$ Use objective evaluation when LAH is suspected (imaging e.g. echocardiography, or invasive measurement using e.g. pulmonary artery catheter)

${ }^{\mathrm{c}}$ Onset of pulmonary symptoms (e.g. hypoxemia-lower $\mathrm{P} / \mathrm{F}$ ratio or $\mathrm{SpO}_{2}$ ) should be within $6 \mathrm{~h}$ of end of transfusion. The additional findings needed to diagnose TRALI (pulmonary edema on a lung imaging study and determination of lack of substantial LAH) would ideally be available at the same time but could be documented up to $24 \mathrm{~h}$ after TRALI onset

${ }^{\mathrm{d}} \mathrm{Use} \mathrm{PaO}_{2} / \mathrm{F}_{\mathrm{i}} \mathrm{O}_{2}$ ratio deterioration along with other respiratory parameters and clinical judgement to determine progression from mild to moderate or severe ARDS. See conversion table in appendix to convert nasal $\mathrm{O}_{2}$ supplementation to $\mathrm{F}_{\mathrm{i}} \mathrm{O}_{2}$

\section{Compliance with ethical standards}

Conflict of interest Both authors declare that they have no conflict of interests.

\section{References}

1. Kleinman S, Caulfield T, Chan P, Davenport R, McFarland J, McPhedran S et al (2004) Toward an understanding of transfusionrelated acute lung injury: statement of a consensus panel. Transfusion 44(12):1774-1789
2. Vlaar APJ, Toy P, Fung M, Looney MR, Juffermans NP, Bux J, Bolton-Maggs P, Peters AL, Silliman CC, Kor DJ, Kleinman S (2019) A consensus redefinition of transfusion-related acute lung injury. Transfusion 59(7):2465-2476. https://doi.org/10.1111/trf.15311

3. Vlaar APJ, Toy P, Fung M, Looney MR, Juffermans NP, Bux J, Bolton-Maggs P, Peters AL, Silliman CC, Kor DJ, Kleinman S (2019) An update of the transfusion-related acute lung injury (TRALI) definition. Transfus Clin Biol. https://doi.org/10.1016/j. tracli.2019.05.007

4. Vlaar A, Kleinman S (2019) An update of the transfusion-related acute lung injury (TRALI) definition. Turk J Haematol. https://doi. org/10.4274/tjh.galenos.2019.2019.0279 
5. Wiersum-Osselton JC, Whitaker B, Grey S, Land K, Perez G, Rajbhandary S, Andrzejewski C Jr, Bolton-Maggs P, Lucero H, Renaudier P, Robillard P, Santos M, Schipperus M (2019) Revised international surveillance case definition of transfusion-associated circulatory overload: a classification agreement validation study.
Lancet Haematol 6(7):e350-e358. https://doi.org/10.1016/S23523026(19)30080-8

Publisher's Note Springer Nature remains neutral with regard to jurisdictional claims in published maps and institutional affiliations. 